\title{
Hyperspectral interferometry for single-shot absolute measurement of 3-D shape and displacement fields
}

\author{
J. M. Huntley, T. Widjanarko, and P. D. Ruiz \\ Loughborough University, Wolfson School of Mechanical and Manufacturing Engineering, \\ Loughborough LE11 3TU, UK
}

\begin{abstract}
We propose a method that we call Hyperspectral Interferometry (HSI) to resolve the $2 \pi$ phase unwrapping problem in the analysis of interferograms recorded with a narrow-band light source. By using a broad-band light source and hyperspectral imaging system, a set of interferograms at different wavenumbers are recorded simultaneously on a high resolution image sensor. These are then assembled to form a three-dimensional intensity distribution. By Fourier transformation along the wavenumber axis, an absolute optical path difference is obtained for each pixel independently of the other pixels in the field of view. As a result, interferograms with spatially distinct regions are analysed as easily as continuous ones. The approach is illustrated with a HSI system to measure 3-D profiles of optically smooth or rough surfaces. Compared to existing profilometers able to measure absolute path differences, the single shot nature of the approach provides greater immunity from environmental disturbance.
\end{abstract}

\section{Introduction}

Interferometry is a well-established optical technique for measuring distances between an object and a reference surface to an accuracy of a small fraction of the wavelength of light. Although sometimes used in a pointwise or linewise configuration, it is at its most powerful when used to provide two-dimensional information. Examples of commercial applications include optical component testing, displacement field measurement using speckle interferometry, and profilometry of small scale mechanical and electronic devices using scanning white light interferometry.

In its standard forms, interferometry can suffer from some drawbacks related firstly to the uniqueness of the measured optical path length data, and secondly to the sensitivity of the technique to environmental disturbances. In the current paper we propose a generic form of 2-D interferometer based on simultaneous illumination of the sample with light of multiple wavelengths, and subsequent separation of the different wavelength bands using a hyperspectral imaging system. The multiple wavelength approach solves the uniqueness problem, and the simultaneous illumination provides a single shot capability that can practically eliminate the influence of environmental disturbance. We describe the concept of hyperspectral interferometry (HSI) in Section 2 before presenting an example of such a hyperspectral interferometer for measuring 3-D surface profiles in Section 3. 


\section{Hyperspectral interferometry}

Consider the general equation for the intensity distribution from a two-beam interferometer:

$$
I(x, y, k)=I_{0}(x, y)+I_{1}(x, y) \cos \left[k z(x, y)+\phi_{0}\right],
$$

where $x$ and $y$ are two image plane coordinates, $z$ is the optical path difference between object and reference waves, $\phi_{0}$ is a phase shift between the waves, $k$ is the wavenumber $2 \pi / \lambda$ where $\lambda$ is the wavelength, and $I_{0}$ and $I_{1}$ are respectively the dc and modulation intensities. Provided a narrow band light source such as a laser is used, Eqn. (1) is applicable to many different classes of two-beam interferometer (e.g. Michelson, Mach-Zehnder, Fizeau etc.) with either smooth or speckled wavefronts.

The usual method of analysing such interferograms is to introduce known phase shifts $\phi_{0}$. However, this only measures the phase due to the optical path difference, $k z(x, y)$, to an integral multiple of $2 \pi$. Objects containing discontinuities (steps, boundaries etc) that result in spatial undersampling of the phase distribution cannot be measured unambiguously.

Hyperspectral interferometry [1] uses an alternative approach. An image is formed by an interferometer using broad band illumination. Unlike the case of a Scanning White Light Interferometer (SWLI) [2], this 'white-light' interferogram is split optically into its constituent interferograms, each formed from a narrow spectral band within the broad spectral illumination envelope, by means of a hyperspectral imaging system. The imaging system places the narrow band images at discrete locations on a two-dimensional photodetector array. If the object is optically smooth, fringes are visible because of the narrow bandwidth of the illumination used for each frame. If the object is optically rough, a speckle field is visible rather than interference fringes, because the starting phase is random. In either case, the phase at a given pixel changes from one sub-image to the next by an amount proportional to the $z$ value for that pixel.

After readout of the array into computer memory, the individual sub-images are registered with respect to their $x$ and $y$ coordinates and stacked to form a sampled hyperspectral image volume $I\left(x_{m}, y_{n}, k_{p}\right)$, in which the third axis specifies the wavenumber (see Fig. 2). Subscripts $m$, $n$ and $p$ take the values $m=0,1,2, \ldots, N_{x}-1, n=0,1,2, \ldots, N_{y}-1$, and $p=0,1,2, \ldots, N_{k}-1$, respectively, where $N_{x}, N_{y}$ and $N_{k}$ are the number of sample points along the respective axes.

According to Eqn. (1), the measured intensity for given location $\left(x_{m}, y_{n}\right)$ then varies cosinusoidally with $k$, with angular frequency $z=z_{0}\left(x_{m}, y_{n}\right)$. The frequency can be measured by performing a 1-D Fourier transform of the intensity values $I\left(x_{m}, y_{n}, k\right)$ with respect to $k$, after subtraction of the mean value, and searching for the peak of the transform which is located at $z$ $=z_{0}\left(x_{m}, y_{n}\right)$. This process is repeated at all the $\left(x_{m}, y_{n}\right)$ pixel locations, thereby providing absolute two-dimensional optical path difference distributions from the single shot measurement. The additional information provided by the $k$ axis allows reliable determination of the absolute path length even though the field of view may contain spatially separated regions, and which would therefore be impossible to unwrap correctly from a single narrow band image alone.

\section{Application to 3-D shape measurement}

One application of the proposed approach is for measuring the 3-D profile of small-scale components. Currently the technique finding most favour in commercial systems is SWLI because of its ability to measure discontinuous objects unambiguously, and its excellent height resolution. However, the technique suffers from vibration artefacts due to the time to perform the scan and requires the use of expensive anti-vibration tables.

The short exposure time of the HSI approach proposed here on the other hand provides a potential solution to making dynamic interferometric measurements. A previously-described 
interferometric profilometer proposed by Schwider and Zhou [3] also acquires data in a single shot, but only provides 1-D information (i.e., profile along a line) rather than the single-shot area scan offered by the instrument proposed here.

A system to demonstrate the proof of principle of the technique is shown schematically in Fig. 1. Light from 2 sources, denoted $\mathrm{LS}_{1}$ and $\mathrm{LS}_{2}$, is combined by a fibre coupler, FC. $\mathrm{LS}_{1}$ is a broadband superluminescent LED (SLED) light source (Superlum Diodes Ltd., 840 HP1) with centre wavelength $840 \mathrm{~nm}$, and full width half maximum $50 \mathrm{~nm} . \mathrm{LS}_{2}$ is a narrowband source (HeNe laser; wavelength $633 \mathrm{~nm}$ ) which is used for alignment purposes. The output from the optical fibre passes first through an etalon, E, is next collimated by lens $\mathrm{L}_{1}$ and then enters a Linnik interferometer head comprising beam splitter $\mathrm{BS}_{1}$, lenses $\mathrm{L}_{2}$ and $\mathrm{L}_{3}$, and reference mirror RM. The sample $\mathrm{S}$ is mounted on a translation stage, TS, to allow the region of interest to be adjusted.

Light from a single point $\mathrm{P}$ on $\mathrm{S}$ produces a bundle of parallel rays that enter the hyperspectral imaging system that comprises beam splitter $\mathrm{BS}_{2}$, diffraction grating, $\mathrm{G}$, and digital camera, $\mathrm{C}_{1}$. The blazed grating is arranged in the Littrow configuration to maximize diffraction efficiency. The parallel rays are brought to a focus in the plane of the photodetector array of $\mathrm{C}_{1}$. Without $\mathrm{E}$ in place, the effect of the grating is to smear the image of $\mathrm{P}$ along a line on $\mathrm{C}_{1}$. The etalon, which has a specified free spectral range of $0.5 \mathrm{~nm}$ at $840 \mathrm{~nm}$ and a finesse $>15$, modifies the spectral content of the illuminating beam to produce a broadband comb with uniform wavenumber spacing, and thus produces instead a set of $N_{k}$ discrete spots. Points near to $\mathrm{P}$ on the sample are in turn imaged onto a set of $N_{k}$ neighbouring points on $\mathrm{C}_{1}$. Thus a set of $N_{k}$ images of the sample are replicated across the sensor array of camera $\mathrm{C}_{1}$, with a small wavenumber shift from one image to the next. The second camera, $\mathrm{C}_{2}$, and imaging lens $\mathrm{L}_{5}$, are used to produce a single high-resolution broad-band image of the object to assist in alignment of the optical system.

An example of the intensity distribution from $\mathrm{C}_{1}$ for a stepped sample consisting of an aluminized microscope glass cover slip mounted on a planar aluminized glass substrate is shown in Fig. 2, in which 62 sub-images of $11 \times 19$ pixels each are formed within a horizontal band. Three such sub-images are shown magnified as in insert to Fig. 2. The step is visible as a vertical discontinuity in the fringe patterns passing approximately through the centre of the field of view. The presence of the discontinuity causes a relative shift of the fringes with changing $k$, which is clearly visible in these three sub-images.

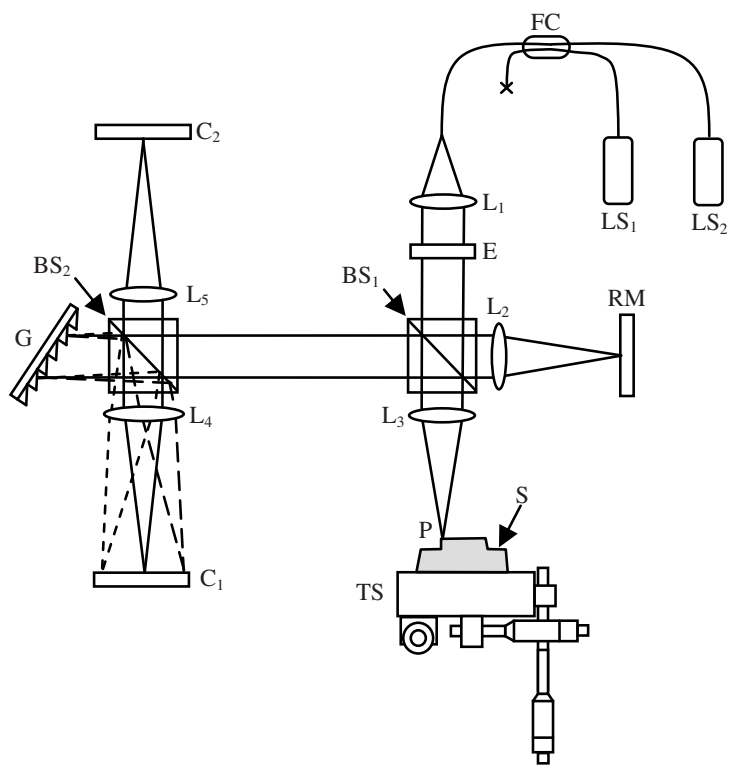

Fig. 1. Hyperspectral interferometer for single-shot 3-D shape measurement. 


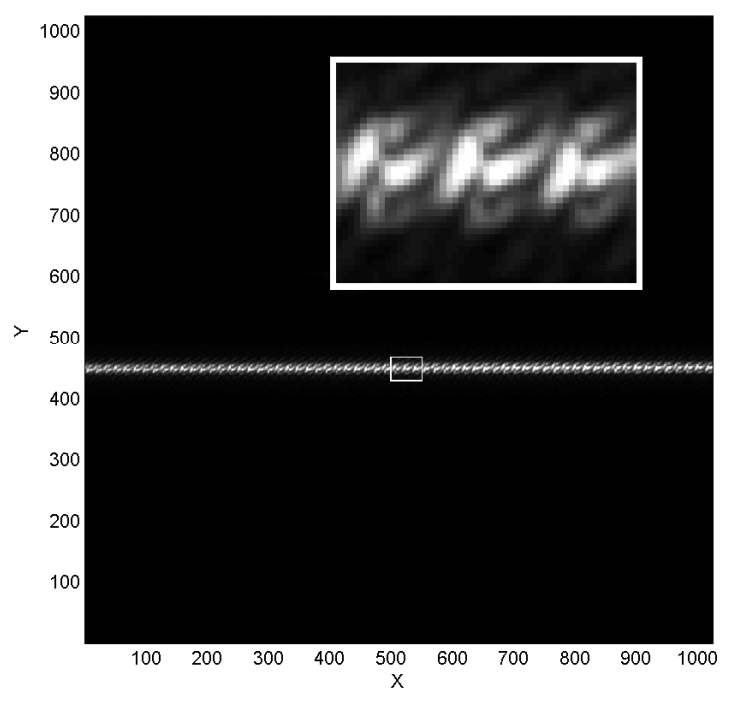

Fig. 2. Set of $N_{k}=62$ hyperspectral images of a stepped surface spread across the horizontal axis of camera $\mathrm{C}_{1}$. Inset: three of the hyperspectral images within the central white box enlarged by a factor $10 \times$.

Sample data from a single pixel at the centre of the field of view of a planar sample is shown in Fig. 3. Parts (a), (b) and (c) were extracted from three full hyperspectral images, each recorded at a different distance from the plane of zero optical path difference. There is significant variation with $k$ in both the fringe modulation and the dc offset due to the non-uniform spectrum of the SLED light source. Removal of the dc 'pedestal' is achieved by recording separate hyperspectral image volumes of the object wave and reference waves, denoted by $I_{o}(x, y, k)$ and $I_{r}(x, y, k)$, respectively, and subtracting these from the recorded interferograms. The procedure to remove the DC pedestal is given in detail in [1].

After Fourier transformation along the $k$ axis, the approximate noise in the measurements was estimated by fitting a plane to each of the measured surfaces. This gave an overall rms for the three planes of $81 \mathrm{~nm}$. This figure is higher than the values of order $1 \mathrm{~nm}$ (or less) typically achieved by SWLI. There are several reasons for this which may be summarized as follows. Firstly, the bandwidth of the light source actually used for the data acquisition was $30 \mathrm{~nm}$ which is an order of magnitude lower than that typically used in SWLI. Depth resolution scales inversely with bandwidth so one can expect a factor of $10 \times$ worse performance due to this factor alone. Secondly spatial variations in photodetector sensitivity have not been taken into account so far. These variations will introduce apparent noise in the measured profiles in the case of hyperspectral interferometry, but not for SWLI, because in the latter case the same pixel provides all the data for a given point on the sample. This effect could be significantly reduced by appropriate calibration of the image sensor.

Finally, the result of analysing the interferogram of the stepped sample (Fig. 2) is shown in Fig. 4. The stepped surface that fell within the illuminated part of the sample is reproduced in the computed 3-D profile. The calculated step height of $115 \mu \mathrm{m}$ agrees well with the true value of 120 $\mu \mathrm{m}$.

Future work will involve extending the application of the technique to the measurement of depth-resolved displacement fields in weakly-scattering media. The data volumes produced by HSI are essentially equivalent to those produced by phase contrast wavelength scanning interferometry, so that the well-established analysis procedures described for example in [4] could equally well be applied to the data produced by HSI. 
(a)

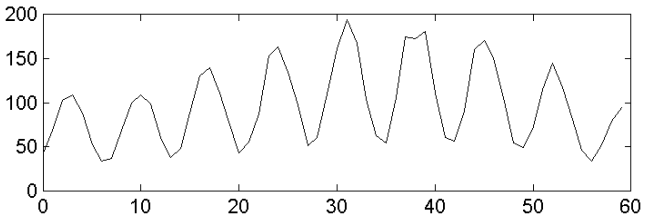

(b)

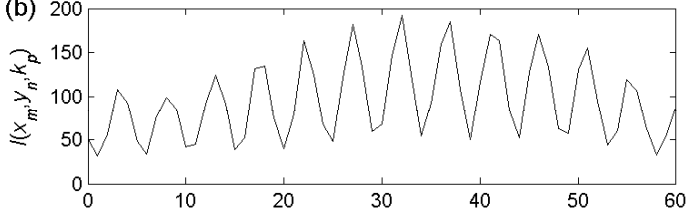

(c)

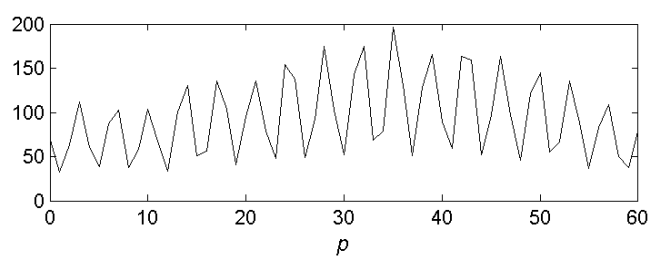

Fig. 3. 1-D signal $I\left(x_{m}, y_{n}, k_{p}\right)$ from a pixel $\left(x_{m}, y_{n}\right)$ close to the centre of the field of view for the planar sample at three values (increasing from (a) to (c)) of optical path difference.

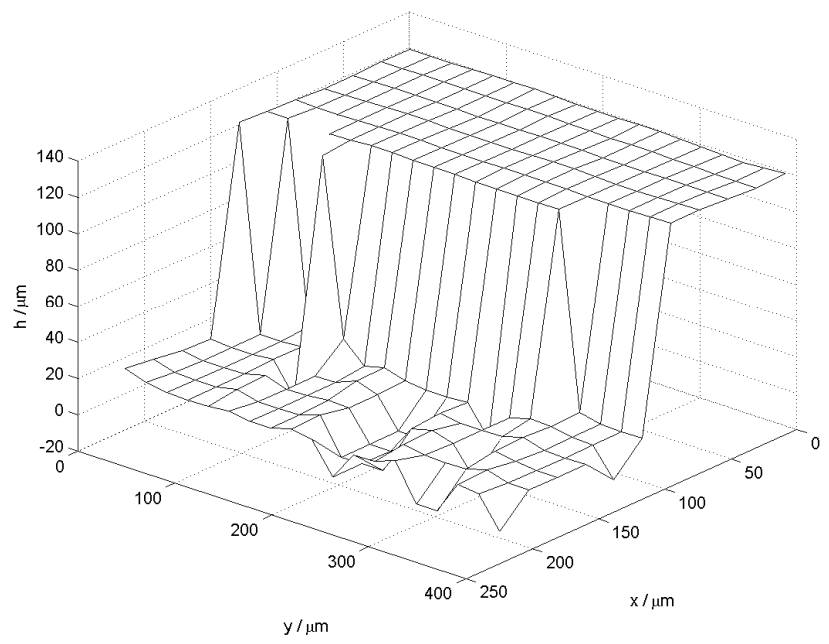

Fig. 4. Surface profile of the stepped sample on a $11 \times 19$ pixel grid measured using the single-shot HSI system. 


\section{Conclusions}

A new interferometric approach to the problem of determining 2-D absolute optical path differences has been proposed in this paper. Unlike current methods, such as scanning white light interferometry and wavelength scanning interferometry, the data are acquired in a single shot, thereby providing significantly greater immunity from environmental disturbance. Proof of principle experiments with a 'white light' interferometer have demonstrated the applicability of the technique to the single-shot measurement of absolute 3-D profiles. An unambiguous depth measurement range of $350 \mu \mathrm{m}$ and a depth measurement precision of approximately $80 \mathrm{~nm}(=1$ part in 4,000 of the depth range) were achieved with a bandwidth of $30 \mathrm{~nm}$ and a centre wavelength of $840 \mathrm{~nm}$.

\section{References}

1. J. M. Huntley, T. Widjanarko, and P. D. Ruiz, Meas. Sci. Technol. (submitted Feb. 2010).

2. L. Deck and P. de Groot, Appl. Opt. 33, 7334 (1994).

3. J. Schwider and L. Zhou, Opt. Lett. 19, 995 (1994).

4. P. D. Ruiz, J. M. Huntley and R. D. Wildman, Appl. Opt. 44, 3945 (2005). 